Results We identified 15 patients currently receiving BEL therapy. Of these, 9 were not analysed further because they had no history of LN or NPSLE.

One 48-y/o female patient after renal transplantation with background therapy consisting of prednisone (GC), hydroxychloroquine (HCQ), leflunomide (LEF) and tacrolimus (TAC) had a stable disease but no additional benefit (BEL stopped after 5 months). Three female patients with GC, HCQ and mycophenolate mofetil (MMF) had an improvement of proteinuria, steroid dosage and overall quality of life. One female patient is receiving BEL or placebo (PBO) during a clinical trial (BLISS-LN), she has markedly improved proteinuria with GC, HCQ, MMF and BEL/PBO. One 73-y/o male patient with NPSLE who failed or could not tolerate various standard and additional therapies (including Rituximab and Cyclophosphamide) had a persistent clinical improvement of cutaneous lupus and neuropsychiatric symptoms (dysarthria, concentration, ataxia) after the second BEL infusion. Overall, there was one upper respiratory tract infection but no other adverse events.

Conclusions In the six patients analysed, 3 had improved proteinuria, 1 had stable disease after renal transplantation, 1 improved regarding NPSLE symptoms and 1 had improved proteinuria, but in the last case, it is not yet clear whether the effect is due to BEL. Overall, while the results of the BLISS-LN trial are awaited, we experienced improved Lupus nephritis with BEL in addition to standard therapy and observed one case of improved NPSLE. While BEL has not been approved for these severe organ manifestations, it still might be effective in well-selected patients.

\section{PS8:168 PROTEINURIA IN RELATION TO CLASS OF LUPUS NEPHRITIS - A RETROSPECTIVE SINGLE-CENTRE STUDY}

LE Schrempf, J-G Rademacher, M Plüß, GA Müller, P Korsten. University Medical Centre Göttingen, Department of Nephrology and Rheumatology, Göttingen, Germany

\subsection{6/lupus-2018-abstract.211}

Purpose Lupus nephritis (LN) is one of the most severe organ complications of Systemic lupus erythematosus (SLE) affecting up to $60 \%$ throughout the course of their disease. Currently, LN is classified according to the ISN/RPS classification. Classes III/IV require aggressive immunosuppressive treatment to avoid end-stage renal disease. However, there are no clinical or serological parameters to predict the type of renal disease and overall renal prognosis.

Methods We performed a single-centre study at our institution of all patients who underwent a renal biopsy between 2001 and 2017. Proteinuria, creatinine and other clinical/serological data were collected. Median values were analysed with ANOVA and Bonferroni's correction for multiple comparisons. Results 49 patients were analysed in our study. 3 patients were excluded because of incomplete data. The remaining 46 patients were stratified according to the histopathological class of Lupus nephritis. 2 patients had class I, 7 patients had class II, 12 patients had class III, 2 patients had class III/V, 15 patients had class IV, 2 patients had class IV/V and 6 patients had pure class $\mathrm{V}$.

Median proteinuria at or around the nearest time point to renal biopsy were $1487 \mathrm{mg} / \mathrm{g}$ creatinine (Cr) (class I), $1515 \mathrm{mg} / \mathrm{g}$ Cr (class II), $1373 \mathrm{mg} / \mathrm{g} \mathrm{Cr}$ (class III), $3528 \mathrm{mg} / \mathrm{g}$
Cr (class III/V), $3190 \mathrm{mg} / \mathrm{g}$ Cr (class IV), $5741 \mathrm{mg} / \mathrm{g} \mathrm{Cr}$ (class IV/V) and $1773 \mathrm{mg} / \mathrm{g} \mathrm{Cr}$ (class V).

While LN classes III/V, IV and IV/V showed the highest median proteinuria, there was no statistical difference between groups.

Conclusions Although often presumed, proteinuria is not a reliable marker for the various types of Lupus nephritis. There was a higher median proteinuria with class $\mathrm{V}$ (pure or combined) membranous nephropathy, however, even proteinuria in this group was not significantly different compared with the other groups. Lack of reliable clinical markers challenges the current lupus nephritis classification system, a combination of clinical, serological and histopathological findings might more appropriately predict the overall prognosis in LN.

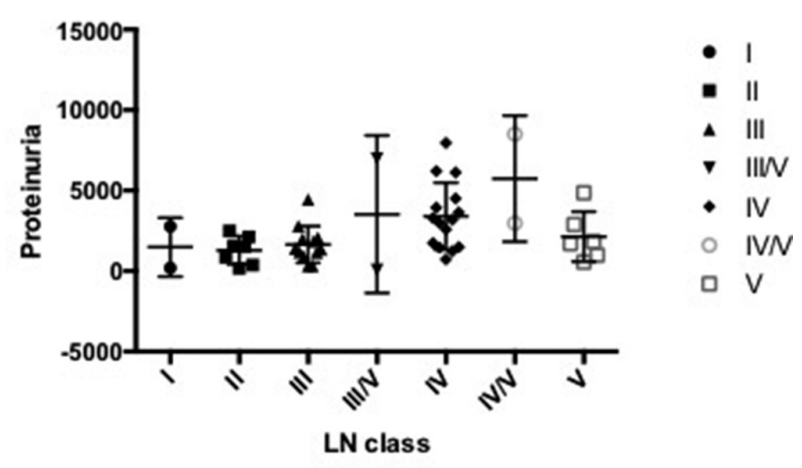

Abstract PS8:168 Figure 1

\section{PS8:169 PREVALENCE AND IMPACT OF DYSLIPIDEMIA IN LUPUS NEPHRITIS PATIENTS}

I Haddiya. Faculty of Medicine, University Mohamed First, Oujda, Morocco

10.1136/lupus-2018-abstract.212

Dyslipidemia is a well-established atherosclerotic risk factor. It is also believed to affect the outcome of SLE, especially in lupus nephritis patients (LN). The aim of this study was to assess the prevalence and impact of dyslipidemia in our LN patients.

Methods We performed a retrospective clinical study, 140 patients with biopsy-proven LN from were analysed. The renal activity and classification were evaluated according to renal pathology. SLE disease activity was scored using the SLE Disease Activity Index (SLEDAI). Adverse outcome was defined by the occurrence of ESRD or death. The correlations between dyslipidemia and both ESRD and mortality were assessed.

Results Mean age of our patients was $34.63 \pm 12,7$ years old, $83 \%$ were females. Class III, IV and V lupus nephritis accounted for $21 \%, 58.7 \%$ and $11.2 \%$ The prevalence of dyslipidemia with elevations in total cholesterol (TC), low-density lipoprotein (LDL), triglyceride (TG) were noted in in our LN patients, ranging from $41 \%$ at diagnosis to $59.7 \%$ or even higher after 24 months, and statitns were administered in $23 \%$ of the patients

After a mean follow-up of 22 months, ESRD occured in $24 \%$, and death in $13 \%$ of cases. Moreover, dyslipidemia was significantly associated to both ESRD $(p<0.02)$ and death $(\mathrm{p}<0.003)$. 